Case Study

\title{
Case study of continuous knee joint traction treatment on the pain and quality of life of patients with degenerative gonarthritis
}

\author{
Dong-Kyu Lee, PT ${ }^{1)}$, NAm-Yong LeE, $\mathrm{PT}^{2)^{*}}$ \\ 1) Department of Physical Therapy, Sunhan Hospital, Republic of Korea \\ 2) Joa-Gym Personal Training Center: 53 Bongseon 2-ro, Nam-gu, Gwangju 61675, Republic of Korea
}

\begin{abstract}
Purpose] The purpose of this study was to examine the effects of continuous knee joint traction treatment on the pain and quality of life of patients with degenerative gonarthritis. [Subjects and Methods] Continuous knee joint traction treatment was administered to 59 year-old man and 62 year-old woman diagnosed with degenerative gonarthritis for 20 minutes, once a day, five times a week for 4 weeks. Pain and quality of life were measured using the visual analogue scale (VAS) and the short form 36-item health survey (SF-36), respectively. [Results] The VAS score of the male patient before treatment was 8 points but it decreased to 1 point after treatment, and SF36 score increased from 81 points before treatment to 93 points after treatment. In addition, the VAS score of the 62 year-old female patient was 7 points before treatment but it decreased to 2 points after treatment, and SF-36 score increased from 78 points before treatment to 96 points after treatment. [Conclusion] The purpose of the study was to report 2 cases of patients with degenerative gonarthritis of the knee who received continuous knee joint traction. Key words: Degenerative gonarthritis, Continuous traction, Pain
\end{abstract}

(This article was submitted Jan. 30, 2018, and was accepted Mar. 15, 2018)

\section{INTRODUCTION}

Degenerative gonarthritis is a disease that results from damage to joint cartilage and the surrounding ligaments and muscles, which are responsible for joint movement ${ }^{1)}$. The joints therefore weaken, and the disease causes severe cartilage abrasion leading to pain, functional disorder, stiffness, reduced range of working, and loss of functional independence ${ }^{1)}$. The persistent pain and functional disorder also leads to sociopsychological issues such as acute depression and declined quality of life ${ }^{2)}$. Studies demonstrated that mechanical joint traction of the knee using an external fixing device on patients with degenerative gonarthritis showed promising results. It increased the joint space and cartilage thickness, decreased the area of lost brain areas, and improved overall function ${ }^{3,4)}$. However, joint traction in previous researches is difficult to apply because they used surgical methods that hampered daily life. The purpose of the study was to report 2 cases of patients with degenerative gonarthritis of the knee who received continuous knee joint traction.

\section{SUBJECTS AND METHODS}

This study is a single case study. The study sample comprised 59 year-old man and 62 year-old woman diagnosed with degenerative gonarthritis, who had no issues of blood pressure, pulse, and breathing. They also had no consciousness or sensory disorders. The study purpose and methods were thoroughly explained to the subjects, and their consent was obtained to carry out the study. The study adhered to the principles of the Declaration of Helsinki. The ethics committee of Nambu University approved this study. The participants were asked to bend their hip and knee joints at 60 degrees in the supine

*Corresponding author. Nam-Yong Lee (E-mail: sweetlny@naver.com)

(C2018 The Society of Physical Therapy Science. Published by IPEC Inc.

(c) (i) $\odot$ This is an open-access article distributed under the terms of the Creative Commons Attribution Non-Commercial No Deriva-

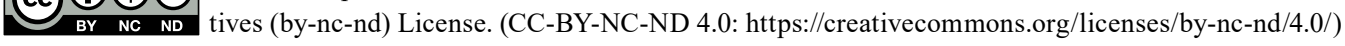


position. The tibia was secured with a strap, and continuous knee joint traction treatment was applied to tow the tibia in the cephalocaudal direction. The force applied by the traction was approximately equal to $6 \%$ of the participant's weight, and traction continued for 20 minutes at a stretch. This treatment was administered for 20 minutes continuously, once a day, and five times a week for 4 weeks. Pain was measured using the visual analogue scale (VAS). The VAS is a self-reported pain scale wherein the patient marks the degree of pain ranging from 0 to 10 , with higher scores indicating more pain. Quality of life was measured using the short form 36-item health survey (SF-36), which consists of eight sections assessed using 36 questions on physical health. The sections are: physical function, limitations of physical roles, pain, general and mental health, vigor, social function, limitations of emotional roles, and mental health. A higher score indicates better quality of life.

\section{RESULTS}

The VAS score of the male patient before treatment was 8 points but decreased to 1 point after treatment, and his SF-36 scores increased from 81 points before treatment to 93 points after treatment. Furthermore, the VAS score of the female patients was 7 points before treatment but decreased to 2 points after, and SF-36 scores increased from 78 points before treatment to 96 points after treatment.

\section{DISCUSSION}

The purpose of the study was to report 2 cases of patients with degenerative gonarthritis of the knee who received continuous knee joint traction. According to the results, pain decreased after applying the continuous traction treatment in this study. An external surgical fixing device used for traction is reported to have positive effects on decreasing pain ${ }^{3)}$. In Alpayci et al. ${ }^{4)}$ study, knee joint traction treatment was found to significantly decrease pain, which was consistent with this study's result. Continuous traction results in muscle relaxation, stimulation of dynamic muscle contractions, and inhibition of protective muscle reflection all of which are instrumental in decreasing pain ${ }^{5}$. Moreover, the increase in the joint space because of continuous knee joint traction is also considered to help reduce pain ${ }^{4}$. The study of Khademi-Kalantari et al. ${ }^{6}$ ) showed that patients' quality of life improved significantly after knee joint traction treatment, which was consistent with the study results. When Alpayci et al. ${ }^{4)}$ administered knee joint traction treatment to patients with degenerative arthritis, their ability to perform physical functions and working range of joints improved. Khademi-Kalantari et al. ${ }^{6}$ ) demonstrated improvement in gait and quality of life of patients with degenerative arthritis after applying knee joint traction treatment. Previous studies have shown that this treatment decreased the patients' pain, increased their ability to perform physical function and working range of joints, and improved gait. Therefore, their quality of life improved simultaneously. There are a few limitations to this study. First, it is difficult to generalize the study results because of the small number of participants. Further, this study could not conduct a follow-up research on how long the effect of continuous knee joint traction lasted after the termination of treatment. To generalize the study results, it will be necessary to conduct a long-term study and follow-up research using a larger study sample.

\section{Conflict of interest}

None.

\section{REFERENCES}

1) Aspden RM: Osteoarthritis: a problem of growth not decay? Rheumatology (Oxford), 2008, 47: 1452-1460. [Medline] [CrossRef]

2) Turner JA, Ersek M, Kemp C: Self-efficacy for managing pain is associated with disability, depression, and pain coping among retirement community residents with chronic pain. J Pain, 2005, 6: 471-479. [Medline] [CrossRef]

3) Intema F, Van Roermund PM, Marijnissen AC, et al.: Tissue structure modification in knee osteoarthritis by use of joint distraction: an open 1-year pilot study. Ann Rheum Dis, 2011, 70: 1441-1446. [Medline] [CrossRef]

4) Alpayci M, Ozkan Y, Yazmalar L, et al.: A randomized controlled trial on the efficacy of intermittent and continuous traction for patients with knee osteoarthritis. Clin Rehabil, 2013, 27: 347-354. [Medline] [CrossRef]

5) Kisner C, Colby LA: Therapeutic exercise: Foundations and techniques, 3rd ed. Philadelphia: F.A. Davis Co., 1996.

6) Khademi-Kalantari K, Mahmoodi Aghdam S, Akbarzadeh Baghban A, et al.: Effects of non-surgical joint distraction in the treatment of severe knee osteoarthritis. J Bodyw Mov Ther, 2014, 18: 533-539. [Medline] [CrossRef] 